\title{
The PLC-based Industrial Temperature Control System: Design and Implementation
}

\author{
Fanjie Wei \\ Chongqing College of Electronic Engineering, No. 76, Eastern Road, Daxuecheng, Shapingba District, Chongqing, 401331, China
}

\begin{abstract}
Targeting at the problem of slow response and low accuracy of the automatic temperature control system for material processing and boiler heating, a new design method is proposed to work with the PLC-based temperature control system, where the box temperature control may be achieved through the fan and the heating plate. The hardware design and software design of the system are analyzed in detail. In this paper, a combination of the traditional PID control and the more popular fuzzy control is taken as the control program to achieve the overall design of the control algorithm. Followed by the simulation in the MATLAB software, the designed system is highlighted by its the characteristics of impressive stability, precision and robustness.
\end{abstract}

Keywords. PLC, temperature control, PID algorithm.

\section{Introduction}

The modern sensing technology and control methods are undergoing continuous innovation, where the real-time temperature control is demanding higher accuracy and faster response more than ever. Temperature control is widely used in production and industrial control processes in all aspects. For example, in the iron and steel smelting process, iron and steel to be baked requires heat treatment in order to achieve their performance indicators; plastic qualitative process also needs to maintain a certain temperature range. The fact is that the temperature control system is a complex process object involving large inertia and pure delay with multi-variable and time-varying parameters. At present, the PID control methodology is adopted in most cases [1]. In this way, different PID parameters should be selected for different control objects, for which some practical experience is needed. As a language controller, the fuzzy PID control is to imitate the way of human thinking and experience to achieve its control process that can more closely reflect the best control behavior of the controller. With strong robustness and control stability, it can be applied to different control objects. The combination of fuzzy control and PLC, which is widely used in industrial control, is one of the hotspots in this research area. Therefore, this work involved the use of the PLC-based fuzzy PID control technology, by which the system temperature was set through the fan and the heating plate to control the box temperature.

\section{System design}

In this design, the temperature control system consists of hardware and software components.

\subsection{System hardware}

In the hardware part of the system, the acquisition module uses the temperature sensor to measure the measured object temperature, and the temperature signals are converted into electrical signals [2], which are then transmitted to the 
temperature transmitter, where the electrical signal is converted into a $4 \sim 20 \mathrm{~mA}$ current signal, so that the module EM235 in the PLC expansion module can be facilitated as to the analog signal input. EM235 receives data, which will be sent directly to the PLC output control text display (display temperature) and the temperature control device [3] (heating \&cooling device). The system block diagram is shown in Figure 1.

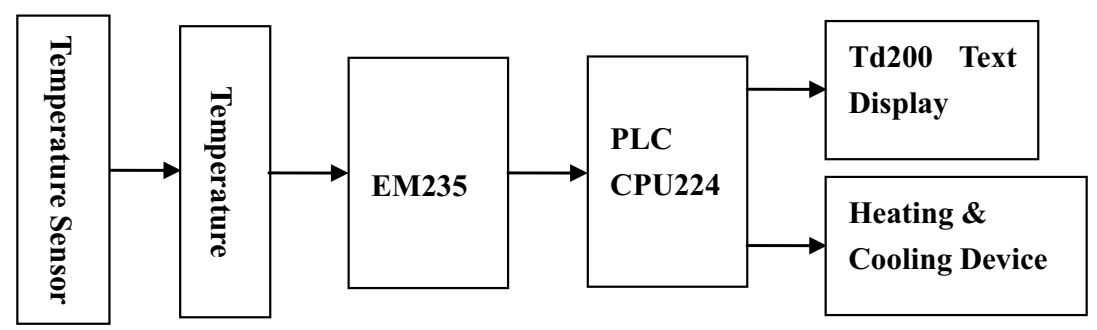

Fig.1. Block Diagram of Temperature Control System.

\subsection{System software}

The software of this system adopts STEP7 for PLC200, the popular programming software by Siemens, for software compilation [4], and the temperature controller device adopts fuzzy PID algorithm for temperature control, with the simulation to be implemented by MATLAB simulation software.

Figure 1 shows that the system consists of four modules, i.e., acquisition module, control module, display module and implementation module. The acquisition module includes PT100 temperature sensor and temperature transmitter. PT100 temperature sensor works with a temperature variable that can be converted into a standardized output signal. This instrument is mainly used for industrial process with measurement temperature and control parameters. The temperature transmitter is a signal conversion device, which is responsible for the signals collected by the temperature sensor to be converted to electrical signals of $4 \sim 20 \mathrm{~mA}$. This is quite convenient for PLC200's identification and collection of temperature signal. For control module, Siemens PLC200 is chosen as the core controller, playing the role of the completion of the temperature signal collection, signal processing and signal transmission. Display module using TD200 text display can be better compatible with the PLC to complete the data transmission. This display module displays the current temperature and the desired temperature. Implementation module works by using cold fan and heating resistor, through the PLC-controlled cold air fan and heating resistor to complete the instructions for the temperature rise or fall, and finally, the temperature reaches a constant value.

\section{Fuzzy PID control technology}

Fuzzy controller is a kind of new controller developed in recent years. The advantage of this device is that it does not require the precise mathematical model of the controlled object. Rather, the control decision table is organized according to the manual control rules, and then the size of the control variable is determined.

\subsection{Fuzzy PID control: principle and rule}

The CPU queries the corresponding fuzzy control table according to the system deviation (Deviation = Given Value Feedback), and deviation change rate (Deviation Rate = Current Cycle Deviation - Upper Cycle Deviation) to get the setting values of $\mathrm{Kp}, \mathrm{Ki}$ and $\mathrm{Kd}$. Then PID operation is performed.

The formation of fuzzy control rules comes from the experience of the operators or experts with their control of knowledge and experience being made into a number of control decision tables. These rules can be expressed in natural language, only generally to be formalized. The current design of the fuzzy controller is basically used in the fuzzy control process, while the system and the deviation of the set value and its rate of change are taken as a fuzzy input. 
This method can not only guarantee the stability of the system, but also reduce the overshoot and oscillation phenomenon.

\subsection{Fuzzy PID control algorithm}

PID control is very effective for the control of linear time-invariant systems, but not well controlled for nonlinear, time-varying complex systems and systems with unclear models. Remarkably, it is quite easy for the fuzzy controller to gain effectively control of the complex and the model unclear systems. Note that the fuzzy controller is lack of the integral link, so it is difficult to completely eliminate the static difference in the fuzzy control system. Furthermore, in the case of insufficient number of the variable classification, a small oscillation is often found near the equilibrium point. If we combine the two control methods, we can form a fuzzy PID controller with the advantages of both.

There are several methods that can be employed to combine fuzzy technology with the PID control algorithm to form a fuzzy PID controller: One is the use of fuzzy controller that allows the PID controller to get its online self-tuning PID parameters, making a fuzzy self-tuning parameter PID controller; another may be done in a large deviation range, where the proportional control is used, while the fuzzy control is adopted in the small deviation. These two control modes can be switched upon the pre-determined deviation threshold, which constitutes the FUZZY-P dual-mode segment controller. Similarly, multi-mode segmentation control algorithm may be designed to achieve sub-sections according to different conditions and requirements, with different modes of control.

\subsection{Difference between fuzzy control and the traditional PID control}

The distinction between the two may be understood as: PID control is time-based and fuzzy control is a process-based one. The structural difference between the two is shown in Figure 2.

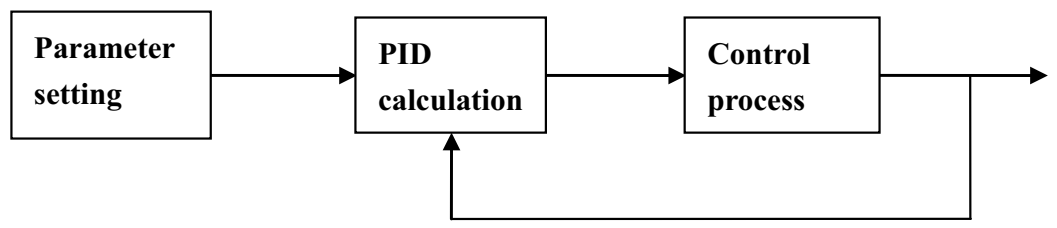

(a)

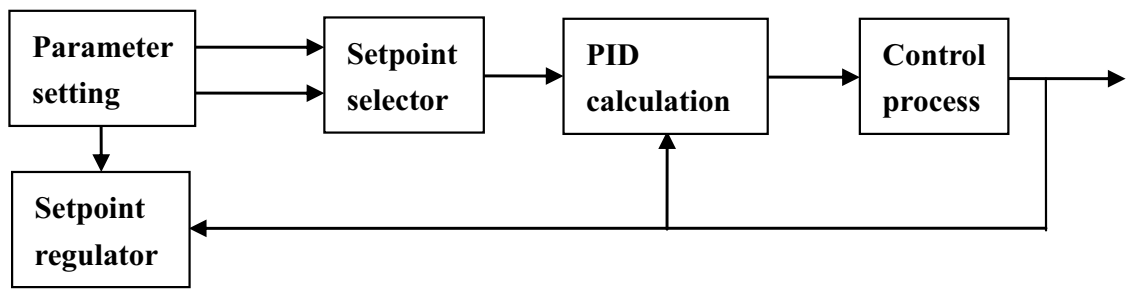

(b)

Fig.2. Difference Between Fuzzy Control and Traditional PID Control.

(a) Traditional PID Control System - Block Diagram (b) Fuzzy PID Control System - Block Diagram

The intelligence of the fuzzy controller, compared to the traditional PID controller, is reflected in an extra setpoint regulator and setpoint selector. The setpoint regulator simulates the control procedures and functions of an experienced operator. It focuses on the control process (in the range of PID control), the dynamic characteristics of the system and the non-linearity (including load changes), and the system is operated by inputting the assumed target value to the PID operator. As the fuzzy control significantly reduces the overshoot, it is capable of effectively improving the system 
response speed and temperature control accuracy, being easier to achieve computer control. For this reason, fuzzy control technology is used by many control systems. Concerning the control of heat treatment equipment, fuzzy control technology marks the most significant progress in the last decade, attracting more and more attention by the heat treatment sector. Fuzzy PID control is present in a series of control applications, including the resistance furnace temperature system, the raw material mixed system, beer fermentation monitoring system, the wind network throttle control system, magnetic levitation system, motor control system, cement cooling process control, air conditioning constant pressure water supply system, radar seeker servo system and so on. This is sufficient to illustrate the excellence of the fuzzy PID control algorithm.

\section{Software design}

According to the system design requirements, the software program flow is shown in Figure 3.

First of all, the parameters of the temperature control system undergoes wake-up initialization, mainly to set the control temperature and the PID initial value, including the value settings of PID gain, PID integral time, PID differential time and PID sampling time. Then, the ambient temperature is collected through the sensor in a range of $6400 \sim 320000$, as the digital signal. As the fuzzy PID algorithm requires real-format temperature signal input, there is the need for $\mathrm{A} / \mathrm{D}$ conversion of temperature signal prior to the PID algorithm process. The collected digital signal is converted into double integer signal, which is then transformed into a real figure. The actual temperature is calculated by the temperature calculation formula. The measured temperature is taken as the input signal for PID operation, and the output is ready for the control of the heating resistance and cold air fan.

In the design, the temperature control is based on the PID control algorithm. PID is the most commonly applied in industrial production, a control method being able to meet the need for high-precision measurement and control systems. Using PID algorithm to achieve the temperature control system can be more stable and reliable [5].

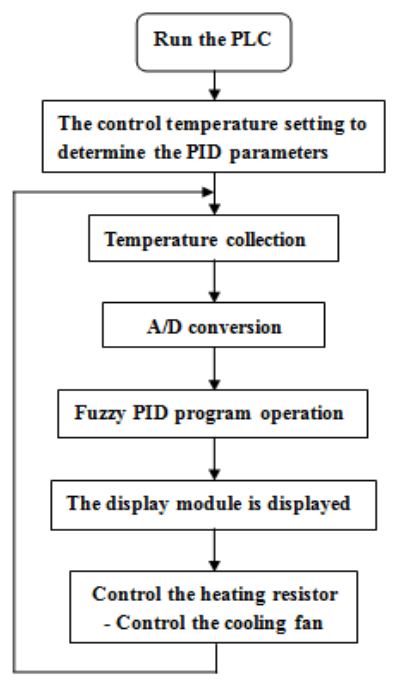

Fig.3. Program Flow.

\section{Experiment and Simulation}

Assume that the system has an open-loop transfer function as $\mathrm{G}(\mathrm{s})=1 / \mathrm{S}(2 * \mathrm{~S}+5)$, and a fuzzy control system is established in Matlab, as shown in Figure 4. At this time, the amplifier Gain $=-1000$, Gain1 $=0.05$, Gain2 $=0.01$. Select the controlled object and its reference model, and we can get the simulation curve shown in Figure 5. 


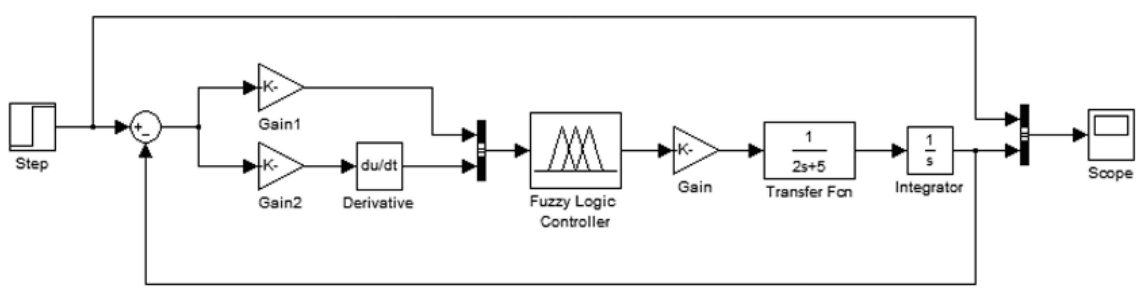

Fig.4. Block Diagram of Fuzzy Control System.

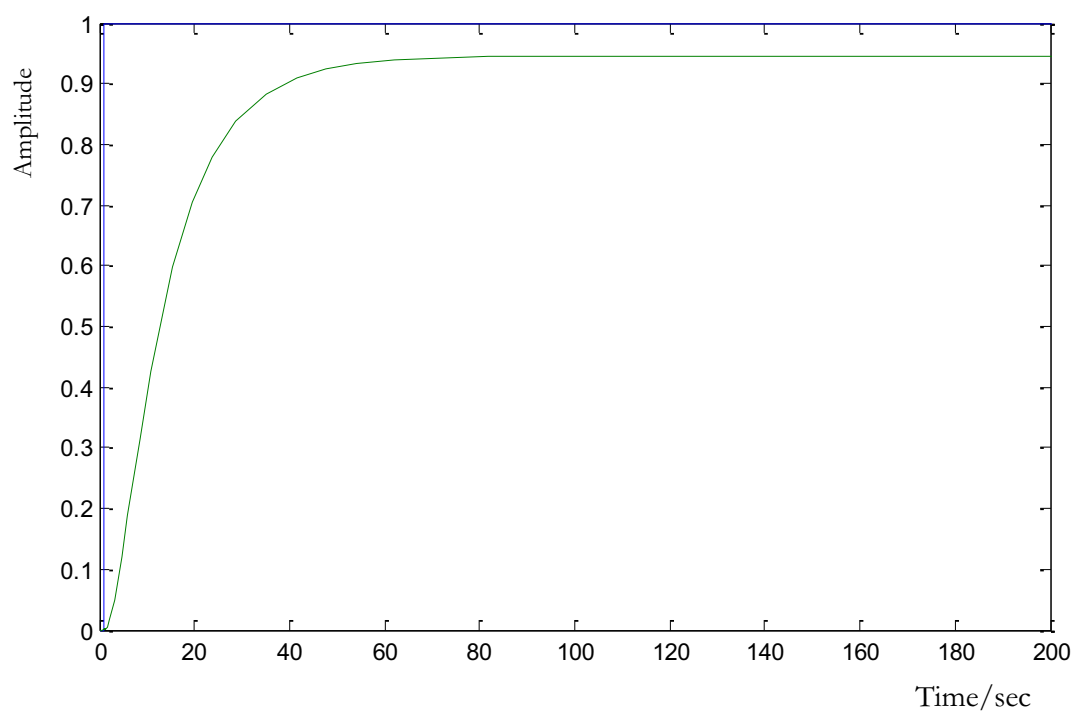

Fig.5. Simulation Results of Fuzzy Control System.

\section{Conclusions}

In this work, the temperature control system was available for real-time display of the temperature inside the box. By setting the temperature through the fan and the heating plate, PID control algorithm was introduced to control the temperature of the box to achieve the temperature control needs. With Siemens PLC200 as the controller, system control was quite impressive with high precision, stability and reliability, being not susceptible to outside interference. On-site commissioning revealed that box temperature could be successfully controlled by the system. This work is expected to bring about a good application prospect.

\section{References}

1. Fang Yanjun. Sun Jian. Intelligent Instrument Technology and its Application. Beijing: Chemical Industry Press .2003: 42-44.

2. Jiang Zhongliang, Chen Xiuyun. Measurement and Control of Temperature. Beijing: Tsinghua University Press, 2005: 26-27.

3. Chai Ruijuan. Chen Haixia. Siemens PLC Programming Technology and Engineering Applications. Machinery Industry Press .2006: 8-14

4. Tang Yifan. Electrical and Programmable Logic Controller Technology. Machinery Industry Press .2004 
5. RenZhicheng. Application of Intelligent Digital Display Controller for Sensors and Transmitters. Beijing: China Electric Power Press .2007: 85-96 